\title{
On Hermite-Hadamard Type Inequalities for $s$-Convex Functions on the Coordinates via Riemann-Liouville Fractional Integrals
}

\author{
Feixiang Chen \\ School of Mathematics and Statistics, Chongqing Three Gorges University, Wanzhou, Chongqing 404000, China \\ Correspondence should be addressed to Feixiang Chen; cfx2002@126.com
}

Received 29 December 2013; Accepted 14 April 2014; Published 30 April 2014

Academic Editor: Fernando Simões

Copyright (C) 2014 Feixiang Chen. This is an open access article distributed under the Creative Commons Attribution License, which permits unrestricted use, distribution, and reproduction in any medium, provided the original work is properly cited.

We obtain some Hermite-Hadamard type inequalities for $s$-convex functions on the coordinates via Riemann-Liouville integrals. Some integral inequalities with the right-hand side of the fractional Hermite-Hadamard type inequality are also established.

\section{Introduction}

If $f: I \rightarrow R$ is a convex function on the interval $I$, then, for any $a, b \in I$ with $a \neq b$, we have the following double inequality:

$$
f\left(\frac{a+b}{2}\right) \leq \frac{1}{b-a} \int_{a}^{b} f(t) d t \leq \frac{f(a)+f(b)}{2} .
$$

This remarkable result is well known in the literature as the Hermite-Hadamard inequality.

Since then, some refinements of the Hermite-Hadamard inequality on convex functions have been extensively investigated by a number of authors (e.g., [1-7]).

Definition 1 (see [8]). Let $s \in(0,1]$ be a fixed real number. A function $f: I \subset[0, \infty) \rightarrow[0, \infty)$ is said to be $s$-convex in the second sense, or that $f$ belongs to the class $K_{s}^{2}$, if the inequality,

$$
f(\alpha x+(1-\alpha) y) \leq \alpha^{s} f(x)+(1-\alpha)^{s} f(y),
$$

holds for all $x, y \in I$ and $\alpha \in[0,1]$.

It can be easily seen that, for $s=1, s$-convexity reduces to ordinary convexity of functions defined on $[0, \infty)$.

In [4], Dragomir defined convex functions on the coordinates as follows.
Let us consider the bidimensional interval $\Delta:=[a, b] \times$ $[c, d]$ in $\mathbb{R}^{2}$ with $a<b$ and $c<d$; a mapping $f: \Delta \rightarrow \mathbb{R}$ is said to be convex on $\Delta$ if the inequality,

$$
\begin{gathered}
f(\lambda x+(1-\lambda) z, \lambda y+(1-\lambda) w) \\
\leq \lambda f(x, y)+(1-\lambda) f(z, w),
\end{gathered}
$$

holds for all $(x, y),(z, w) \in \Delta$ and $\lambda \in[0,1]$

A function $f: \Delta \rightarrow \mathbb{R}$ is said to be coordinated convex on $\Delta$ if the partial mappings $f_{y}:[a, b] \rightarrow \mathbb{R}, f_{y}(u)=f(u, y)$ and $f_{x}:[c, d] \rightarrow \mathbb{R}, f_{x}(v)=f(x, v)$ are convex for all $y \in$ $[c, d]$ and $x \in[a, b]$.

A formal definition for convex functions on the coordinates may be stated as follows.

Definition 2. A function $f: \Delta \rightarrow \mathbb{R}$ is said to be convex on coordinates on $\Delta$ if the inequality,

$$
\begin{aligned}
f(\lambda x & +(1-\lambda) z, t y+(1-t) w) \\
\leq & \lambda t f(x, y)+\lambda(1-t) f(x, w)+(1-\lambda) t f(z, y) \\
& +(1-t)(1-\lambda) f(z, w),
\end{aligned}
$$

holds for all $(x, y),(z, y),(x, w),(z, w) \in \Delta$, and $t, \lambda \in[0,1]$.

In [4], Dragomir established the following Hadamardtype inequalities for convex functions on the coordinates in a rectangle from the plane $\mathbb{R}^{2}$. 
Theorem 3 (see [4]). Suppose that $f: \Delta=[a, b] \times[c, d] \rightarrow \mathbb{R}$ is convex on the coordinates on $\Delta$. Then one has the inequalities:

$$
\begin{aligned}
f\left(\frac{a+b}{2}, \frac{c+d}{2}\right) & \leq \frac{1}{(b-a)(d-c)} \int_{a}^{b} \int_{c}^{d} f(x, y) d y d x \\
& \leq \frac{f(a, c)+f(a, d)+f(b, c)+f(b, d)}{4}
\end{aligned}
$$

The concept of $s$-convex functions on the coordinates in the second sense was introduced by Alomari and Darus in [9].

Let us consider the bidimensional interval $\Delta:=[a, b] \times$ $[c, d]$ in $\mathbb{R}^{2}$ with $a<b$ and $c<d$; a mapping $f: \Delta \rightarrow \mathbb{R}$ is $s$-convex on $\Delta$ if the inequality,

$$
\begin{aligned}
& f(\lambda x+(1-\lambda) z, \lambda y+(1-\lambda) w) \\
& \quad \leq \lambda^{s} f(x, y)+(1-\lambda)^{s} f(z, w),
\end{aligned}
$$

holds for all $(x, y),(z, w) \in \Delta$ with $\lambda \in[0,1]$ and for some fixed $s \in(0,1]$.

A function $f: \Delta \rightarrow \mathbb{R}$ is said to be $s$-convex on the coordinates on $\Delta$ in the second sense if the partial mappings $f_{y}:[a, b] \rightarrow \mathbb{R}, f_{y}(u)=f(u, y)$, and $f_{x}:[c, d] \rightarrow \mathbb{R}$, $f_{x}(v)=f(x, v)$ are $s$-convex in the second sense for all $y \in$ $[c, d]$ and $x \in[a, b]$ with some fixed $s \in(0,1]$.

A formal definition for convex functions on the coordinates in the second sense may be stated as follows.

Definition 4. A function $f: \Delta \rightarrow \mathbb{R}$ is said to be $s$-convex on coordinates in the second sense on $\Delta$ if the inequality,

$$
\begin{aligned}
f(\lambda x & +(1-\lambda) z, t y+(1-t) w) \\
\leq & \lambda^{s} t^{s} f(x, y)+\lambda^{s}(1-t)^{s} f(x, w) \\
& +(1-\lambda)^{s} t^{s} f(z, y)+(1-t)^{s}(1-\lambda)^{s} f(z, w),
\end{aligned}
$$

holds for all $(x, y),(z, y),(x, w),(z, w) \in \Delta$ with $t, \lambda \in[0,1]$ and some fixed $s \in(0,1]$.

In [10], Alomari and Darus proved the following inequalities based on the above definition.

Theorem 5 (see [10]). Suppose that $f: \Delta=[a, b] \times[c, d] \subseteq$ $[0, \infty)^{2} \rightarrow[0, \infty)$ is s-convex on the coordinates in the second sense on $\Delta$. Then one has the inequalities:

$$
\begin{aligned}
4^{s-1} f\left(\frac{a+b}{2}, \frac{c+d}{2}\right) \leq & \frac{1}{(b-a)(d-c)} \int_{a}^{b} \int_{c}^{d} f(x, y) d y d x \\
\leq & (f(a, c)+f(a, d)+f(b, c) \\
& +f(b, d))\left((s+1)^{2}\right)^{-1} .
\end{aligned}
$$

It is remarkable that Sarikaya et al. [11] proved the following interesting inequalities of Hermite-Hadamard type involving Riemann-Liouville fractional integrals.
Theorem 6 (see [11]). Let $f:[a, b] \rightarrow R$ be a positive function with $a<b$ and $f \in L_{1}[a, b]$. If $f$ is a convex function on $[a, b]$, then the following inequalities for fractional integrals hold

$$
f\left(\frac{a+b}{2}\right) \leq \frac{\Gamma(\alpha+1)}{2(b-a)^{\alpha}}\left[J_{a^{+}}^{\alpha} f(b)+J_{b^{-}}^{\alpha} f(a)\right] \leq \frac{f(a)+f(b)}{2},
$$

with $\alpha>0$

We remark that the symbol $J_{a^{+}}^{\alpha}$ and $J_{b^{-}}^{\alpha} f$ denote the leftsided and right-sided Riemann-Liouville fractional integrals of the order $\alpha \geq 0$ with $a \geq 0$ which are defined by

$$
\begin{aligned}
& J_{a^{+}}^{\alpha} f(x)=\frac{1}{\Gamma(\alpha)} \int_{a}^{x}(x-t)^{\alpha-1} f(t) d t, \quad x>a, \\
& J_{b^{-}}^{\alpha} f(x)=\frac{1}{\Gamma(\alpha)} \int_{x}^{b}(t-x)^{\alpha-1} f(t) d t, \quad x<b,
\end{aligned}
$$

respectively. Here, $\Gamma(\alpha)$ is the Gamma function defined by $\Gamma(\alpha)=\int_{0}^{\infty} e^{-t} t^{\alpha-1} d t$

Definition 7 (see [12]). Let $f \in L_{1}[a, b] \times[c, d]$. The RiemannLiouville fractional integrals $J_{a^{+}, c^{+}}^{\alpha, \beta}, J_{a^{+}, d^{-}}^{\alpha, \beta}, J_{b^{-}, c^{+}}^{\alpha, \beta}$ and $J_{b^{-}, d^{-}}^{\alpha, \beta}$ of order $\alpha, \beta>0$ with $a, c \geq 0$ are defined by

$$
\begin{aligned}
& J_{a^{t, c^{+}}}^{\alpha, \beta} f(x, y) \\
& =\frac{1}{\Gamma(\alpha) \Gamma(\beta)} \int_{a}^{x} \int_{c}^{y}(x-t)^{\alpha-1}(y-s)^{\beta-1} f(t, s) d s d t, \\
& x>a, \quad y>c, \\
& J_{a^{+}, d^{-}}^{\alpha, \beta} f(x, y) \\
& \quad=\frac{1}{\Gamma(\alpha) \Gamma(\beta)} \int_{a}^{x} \int_{y}^{d}(x-t)^{\alpha-1}(s-y)^{\beta-1} f(t, s) d s d t, \\
& J_{b^{-}, c^{+}}^{\alpha, \beta} f(x, y) \\
& \quad=\frac{1}{\Gamma(\alpha) \Gamma(\beta)} \int_{x}^{b} \int_{c}^{y}(t-x)^{\alpha-1}(y-s)^{\beta-1} f(t, s) d s d t, \quad y<d, \\
& \quad=\frac{1}{\Gamma(\alpha) \Gamma(\beta)} \int_{x}^{b} \int_{y}^{d}(t-x)^{\alpha-1}(s-y)^{\beta-1} f(t, s) d s d t, \\
& J_{b^{-}, d^{-}}^{\alpha, \beta} f(x, y) \\
& x<b, \quad, y<d .
\end{aligned}
$$

In [12], Sarıkaya proposed the following HermiteHadamard-type inequalities for Riemann-Liouville fractional integrals by using convex functions of two variables on the coordinates. 
Theorem 8 (see [12]). Let $f: \Delta \subseteq \mathbb{R}^{2} \rightarrow \mathbb{R}$ be convex functions on the coordinates on $\Delta=[a, b] \times[c, d]$ in $\mathbb{R}^{2}$ with $0 \leq a<b, 0 \leq c<d$, and $f \in L_{1}(\Delta)$. Then one has the inequalities:

$$
\begin{aligned}
f\left(\frac{a+b}{2}, \frac{c+d}{2}\right) \leq & \frac{\Gamma(\alpha+1) \Gamma(\beta+1)}{4(b-a)^{\alpha}(d-c)^{\beta}} \\
& \times\left[J_{a^{+}, c^{+}}^{\alpha, \beta} f(b, d)+J_{a^{+}, d^{-}}^{\alpha, \beta} f(b, c)\right. \\
& \left.\quad+J_{b^{-}, c^{+}}^{\alpha, \beta} f(a, d)+J_{b^{-}, d^{-}}^{\alpha, \beta} f(a, c)\right] \\
\leq & \frac{f(a, c)+f(a, d)+f(b, c)+f(b, d)}{4} .
\end{aligned}
$$

In [12], Sarıkaya established some Hermite-Hadamard inequalities for convex functions on the coordinates in the second sense via fractional integrals based on the following lemma.

Lemma 9 (see [12]). Let $f: \Delta \subseteq \mathbb{R}^{2} \rightarrow \mathbb{R}$ be a partial differentiable mapping on $\Delta:=[a, b] \times[c, d]$ in $\mathbb{R}^{2}$ with $0 \leq a<b, 0 \leq c<d$. If $\partial^{2} f / \partial t \partial s \in L(\Delta)$, then the following equality holds:

$$
\begin{gathered}
\frac{f(a, c)+f(a, d)+f(b, c)+f(b, d)}{4}+\frac{\Gamma(\alpha+1) \Gamma(\beta+1)}{4(b-a)^{\alpha}(d-c)^{\beta}} \\
\times\left[J_{a^{+}, c^{+}}^{\alpha, \beta} f(b, d)+J_{a^{+}, d^{-}}^{\alpha, \beta} f(b, c)+J_{b^{-}, c^{+}}^{\alpha, \beta} f(a, d)\right. \\
\left.+J_{b^{-}, d^{-}}^{\alpha, \beta} f(a, c)\right]-A \\
=\frac{(b-a)(d-c)}{4} \\
\times\left\{\iint_{0}^{1} t^{\alpha} s^{\beta} \frac{\partial^{2} f}{\partial t \partial s}(t a+(1-t) b, s c+(1-s) d) d s d t\right. \\
\quad-\iint_{0}^{1}(1-t)^{\alpha} s^{\beta} \frac{\partial^{2} f}{\partial t \partial s} \\
\times(t a+(1-t) b, s c+(1-s) d) d s d t \\
\quad-\iint_{0}^{1} t^{\alpha}(1-s)^{\beta} \frac{\partial^{2} f}{\partial t \partial s} \\
\times \iint_{0}^{1}(1-t)^{\alpha}(1-s)^{\beta} \frac{\partial^{2} f}{\partial t \partial s} \\
\times(t a+(1-t) b, s c+(1-s) d) d s d t\}
\end{gathered}
$$

where

$$
\begin{gathered}
A=\frac{\Gamma(\beta+1)}{4(d-c)^{\beta}}\left[J_{c^{+}}^{\beta} f(a, d)+J_{c^{+}}^{\beta} f(b, d)+J_{d^{-}}^{\beta} f(a, c)\right. \\
\left.+J_{d^{-}}^{\beta} f(b, c)\right] \\
+\frac{\Gamma(\alpha+1)}{4(b-a)^{\alpha}}\left[J_{a^{+}}^{\alpha} f(b, c)+J_{a^{+}}^{\alpha} f(b, d)+J_{b^{-}}^{\alpha} f(a, c)\right. \\
\left.+J_{b^{-}}^{\alpha} f(a, d)\right] .
\end{gathered}
$$

In this paper, we establish some Hermite-Hadamard type inequalities for $s$-convex functions on the coordinates functions via Riemann-Liouville integrals. Some integral inequalities with the right-hand side of the fractional HermiteHadamard type inequality are also given.

\section{Fractional Inequalities for $s$-Convex Functions on the Coordinates}

Theorem 10. Suppose that $f: \Delta=[a, b] \times[c, d] \subseteq[0, \infty)^{2} \rightarrow$ $[0, \infty)$ is s-convex function on the coordinates in the second sense on $\Delta$. Then one has the inequalities:

$$
\begin{aligned}
4^{s-1} f & \left(\frac{a+b}{2}, \frac{c+d}{2}\right) \\
\leq & \frac{\Gamma(\alpha+1) \Gamma(\beta+1)}{4(b-a)^{\alpha}(d-c)^{\beta}} \\
& \times\left[J_{a^{+}, c^{+}}^{\alpha, \beta} f(b, d)+J_{a^{+}, d^{-}}^{\alpha, \beta} f(b, c)+J_{b^{-}, c^{+}}^{\alpha, \beta} f(a, d)\right. \\
\leq & \frac{f(a, c)+f(a, d)+f(b, c)+f(b, d)}{4} \\
& \times\left(\frac{1}{(\alpha+s)(\beta+s)}+\frac{1}{\alpha+1} B(\beta, s+1)+\frac{1}{\beta+1}\right. \\
& \quad \times B(\alpha, s+1)+B(\beta, s+1) B(\alpha, s+1)) .
\end{aligned}
$$

Proof. From (7), with $x=t_{1} a+\left(1-t_{1}\right) b, y=\left(1-t_{1}\right) a+t_{1} b$, $z=t_{2} c+\left(1-t_{2}\right) d, w=\left(1-t_{2}\right) c+t_{2} d$, and $t=\lambda=1 / 2$, we get

$$
\begin{aligned}
f\left(\frac{a+b}{2}, \frac{c+d}{2}\right) \leq \frac{1}{4^{s}} & f\left(t_{1} a+\left(1-t_{1}\right) b, t_{2} c+\left(1-t_{2}\right) d\right) \\
+ & f\left(t_{1} a+\left(1-t_{1}\right) b,\left(1-t_{2}\right) c+t_{2} d\right) \\
+ & f\left(\left(1-t_{1}\right) a+t_{1} b, t_{2} c+\left(1-t_{2}\right) d\right) \\
+ & \left.f\left(\left(1-t_{1}\right) a+t_{1} b,\left(1-t_{2}\right) c+t_{2} d\right)\right] .
\end{aligned}
$$


Multiplying both sides of above inequality by $t_{1}^{\alpha-1} t_{2}^{\beta-1}$ then integrating the resulting inequality with respect to $t_{1}, t_{2}$ over $[0,1] \times[0,1]$, we obtain

$$
\begin{gathered}
\frac{1}{\alpha \beta} f\left(\frac{a+b}{2}, \frac{c+d}{2}\right) \\
\leq \frac{1}{4^{s}}\left[\int \int _ { 0 } ^ { 1 } t _ { 1 } ^ { \alpha - 1 } t _ { 2 } ^ { \beta - 1 } f \left(t_{1} a+\left(1-t_{1}\right) b, t_{2} c\right.\right. \\
\left.+\left(1-t_{2}\right) d\right) d t_{2} d t_{1} \\
+\iint_{0}^{1} t_{1}^{\alpha-1} t_{2}^{\beta-1} f\left(t_{1} a+\left(1-t_{1}\right) b,\left(1-t_{2}\right) c\right. \\
+\iint_{0}^{1} t_{1}^{\alpha-1} t_{2}^{\beta-1} f\left(\left(1-t_{1}\right) a+t_{1} b, t_{2} c\right. \\
+\iint_{2}^{1} t_{1}^{\alpha-1} t_{2}^{\beta-1} f\left(\left(1-t_{1}\right) a+t_{1} b,\left(1-t_{2}\right) c\right. \\
\left.\left.+t_{2} d\right) d t_{2} d t_{1}\right] .
\end{gathered}
$$

Using the change of the variable, we get

$$
\begin{aligned}
& \frac{1}{\alpha \beta} f\left(\frac{a+b}{2}, \frac{c+d}{2}\right) \\
& \leq \frac{1}{4^{s}}\left[\int_{a}^{b} \int_{c}^{d}(b-x)^{\alpha-1}(d-y)^{\beta-1} f(x, y) d y d x\right. \\
& \quad+\int_{a}^{b} \int_{c}^{d}(b-x)^{\alpha-1}(y-c)^{\beta-1} f(x, y) d y d x \\
& \quad+\int_{a}^{b} \int_{c}^{d}(x-a)^{\alpha-1}(d-y)^{\beta-1} f(x, y) d y d x \\
& \left.\quad+\int_{a}^{b} \int_{c}^{d}(x-a)^{\alpha-1}(y-c)^{\beta-1} f(x, y) d y d x\right],
\end{aligned}
$$

by which the first inequality is proved. For the proof of the second inequality, we note that $f$ is $s$-convex on coordinates, then

$$
\begin{aligned}
& f\left(t_{1} a+\left(1-t_{1}\right) b, t_{2} c+\left(1-t_{2}\right) d\right) \\
& \leq t_{1}^{s} t_{2}^{s} f(a, c)+t_{1}^{s}\left(1-t_{2}\right)^{s} f(a, d) \\
& \quad+\left(1-t_{1}\right)^{s} t_{2}^{s} f(b, c)+\left(1-t_{1}\right)^{s}\left(1-t_{2}\right)^{s} f(b, d), \\
& f\left(t_{1} a+\left(1-t_{1}\right) b,\left(1-t_{2}\right) c+t_{2} d\right) \\
& \leq t_{1}^{s}\left(1-t_{2}\right)^{s} f(a, c)+t_{1}^{s} t_{2}^{s} f(a, d) \\
& \quad+\left(1-t_{1}\right)^{s}\left(1-t_{2}\right)^{s} f(b, c)+\left(1-t_{1}\right)^{s} t_{2}^{s} f(b, d),
\end{aligned}
$$

$$
\begin{aligned}
& f\left(\left(1-t_{1}\right) a+t_{1} b, t_{2} c+\left(1-t_{2}\right) d\right) \\
& \quad \leq\left(1-t_{1}\right)^{s} t_{2}^{s} f(a, c)+\left(1-t_{1}\right)^{s}\left(1-t_{2}\right)^{s} f(a, d) \\
& \quad+t_{1}^{s} t_{2}^{s} f(b, c)+t_{1}^{s}\left(1-t_{2}\right)^{s} f(b, d), \\
& f\left(\left(1-t_{1}\right) a+t_{1} b,\left(1-t_{2}\right) c+t_{2} d\right) \\
& \leq\left(1-t_{1}\right)^{s}\left(1-t_{2}\right)^{s} f(a, c)+\left(1-t_{1}\right)^{s} t_{2}^{s} f(a, d) \\
& \quad+t_{1}^{s}\left(1-t_{2}\right)^{s} f(b, c)+t_{1}^{s} t_{2}^{s} f(b, d) .
\end{aligned}
$$

Multiplying both sides of above inequalities by $t_{1}^{\alpha-1} t_{2}^{\beta-1}$, then integrating the resulting inequality with respect to $t_{1}, t_{2}$ over $[0,1] \times[0,1]$, we obtain

$$
\begin{aligned}
& \iint_{0}^{1} t_{1}^{\alpha-1} t_{2}^{\beta-1}\left[f\left(t_{1} a+\left(1-t_{1}\right) b, t_{2} c+\left(1-t_{2}\right) d\right)\right. \\
& +f\left(t_{1} a+\left(1-t_{1}\right) b,\left(1-t_{2}\right) c+t_{2} d\right) \\
& +f\left(\left(1-t_{1}\right) a+t_{1} b, t_{2} c+\left(1-t_{2}\right) d\right) \\
& +f\left(\left(1-t_{1}\right) a\right. \\
& \left.\left.+t_{1} b,\left(1-t_{2}\right) c+t_{2} d\right)\right] d t_{2} d t_{1} \\
& \leq[f(a, c)+f(a, d)+f(b, c)+f(b, d)] \\
& \times \iint_{0}^{1} t_{1}^{\alpha-1} t_{2}^{\beta-1}\left[t_{1}^{s} t_{2}^{s}+t_{1}^{s}\left(1-t_{2}\right)^{s}+\left(1-t_{1}\right)^{s} t_{2}^{s}\right. \\
& \left.+\left(1-t_{1}\right)^{s}\left(1-t_{2}\right)^{s}\right] d t_{2} d t_{1} \\
& =[f(a, c)+f(a, d)+f(b, c)+f(b, d)] \\
& \times\left(\frac{1}{(\alpha+s)(\beta+s)}+\frac{1}{\alpha+1} B(\beta, s+1)\right. \\
& \left.+\frac{1}{\beta+1} B(\alpha, s+1)+B(\beta, s+1) B(\alpha, s+1)\right) .
\end{aligned}
$$

Here, using the change of the variable, we have

$$
\begin{gathered}
\frac{\Gamma(\alpha+1) \Gamma(\beta+1)}{(b-a)^{\alpha}(d-c)^{\beta}}\left[J_{a^{+}, c^{+}}^{\alpha, \beta} f(b, d)+J_{a^{+}, d^{-}}^{\alpha, \beta} f(b, c)\right. \\
\left.+J_{b^{-}, c^{+}}^{\alpha, \beta} f(a, d)+J_{b^{-}, d^{-}}^{\alpha, \beta} f(a, c)\right] \\
\leq[f(a, c)+f(a, d)+f(b, c)+f(b, d)] \\
\quad \times\left(\frac{1}{(\alpha+s)(\beta+s)}+\frac{1}{\alpha+1} B(\beta, s+1)\right. \\
\left.\quad+\frac{1}{\beta+1} B(\alpha, s+1)+B(\beta, s+1) B(\alpha, s+1)\right) .
\end{gathered}
$$

The proof is completed. 
Remark 11. Applying Theorem 10 for $s=1$, we get Theorem 8 . We note that the Beta functions is defined by

$$
B(x, y)=\int_{0}^{1}(1-t)^{y-1} t^{x-1} d t, \quad x>0, y>0 .
$$

\section{Inequalities for Differentiable Functions}

Theorem 12. Let $f: \Delta=[a, b] \times[c, d] \subseteq[0, \infty)^{2} \rightarrow[0, \infty)$ be a partial differentiable mapping with $0 \leq a<b, 0 \leq c<d$. If $\left|\partial^{2} f / \partial t_{1} \partial t_{2}\right|$ is s-convex on the coordinates in the second sense on $\Delta$ for some fixed $s \in(0,1]$. Then one has the inequalities:

$$
\begin{aligned}
& \mid \frac{f(a, c)+f(a, d)+f(b, c)+f(b, d)}{4}+\frac{\Gamma(\alpha+1) \Gamma(\beta+1)}{4(b-a)^{\alpha}(d-c)^{\beta}} \\
& \times\left[J_{a^{+}, c^{+}}^{\alpha, \beta} f(b, d)+J_{a^{+}, d^{-}}^{\alpha, \beta} f(b, c)\right. \\
& \left.\quad+J_{b^{-}, c^{+}}^{\alpha, \beta} f(a, d)+J_{b^{-}, d^{-}}^{\alpha, \beta} f(a, c)\right]-A \mid \\
& =\frac{(b-a)(d-c)}{4}\left[\frac{1}{\alpha+s+1}+B(s+1, \alpha+1)\right] \\
& \times\left[\frac{1}{\beta+s+1}+B(s+1, \beta+1)\right] \\
& \times\left[\left|\frac{\partial^{2} f}{\partial t_{1} \partial t_{2}}(a, c)\right|+\left|\frac{\partial^{2} f}{\partial t_{1} \partial t_{2}}(b, c)\right|\right. \\
& \left.\quad+\left|\frac{\partial^{2} f}{\partial t_{1} \partial t_{2}}(a, d)\right|+\left|\frac{\partial^{2} f}{\partial t_{1} \partial t_{2}}(b, d)\right|\right],
\end{aligned}
$$

where $A$ is as given in Lemma 9.

Proof. From Lemma 9, we obtain

$$
\begin{aligned}
& \mid \frac{f(a, c)+f(a, d)+f(b, c)+f(b, d)}{4} \\
& +\frac{\Gamma(\alpha+1) \Gamma(\beta+1)}{4(b-a)^{\alpha}(d-c)^{\beta}}\left[J_{a^{+}, c^{+}}^{\alpha, \beta} f(b, d)+J_{a^{+}, d^{-}}^{\alpha, \beta} f(b, c)\right. \\
& +J_{b^{-}, c^{+}}^{\alpha, \beta} f(a, d) \\
& \left.+J_{b^{-}, d^{-}}^{\alpha, \beta} f(a, c)\right]-A \\
& \leq \frac{(b-a)(d-c)}{4} \\
& \times\left\{\iint_{0}^{1} t_{1}^{\alpha} t_{2}^{\beta} \mid \frac{\partial^{2} f}{\partial t_{1} \partial t_{2}}\left(t_{1} a+\left(1-t_{1}\right) b, t_{2} c\right.\right. \\
& \left.+\left(1-t_{2}\right) d\right) \mid d t_{2} d t_{1}
\end{aligned}
$$

$$
\begin{aligned}
& +\iint_{0}^{1}\left(1-t_{1}\right)^{\alpha} t_{2}^{\beta} \\
& \quad \times \mid \frac{\partial^{2} f}{\partial t_{1} \partial t_{2}} \\
& \quad \times\left(t_{1} a+\left(1-t_{1}\right) b, t_{2} c+\left(1-t_{2}\right) d\right) \mid d t_{2} d t_{1} \\
& +\iint_{0}^{1} t_{1}^{\alpha}\left(1-t_{2}\right)^{\beta} \\
& \quad \times \mid \frac{\partial^{2} f}{\partial t_{1} \partial t_{2}} \\
& \quad \times\left(t_{1} a+\left(1-t_{1}\right) b, t_{2} c+\left(1-t_{2}\right) d\right) \mid d t_{2} d t_{1} \\
& +\iint_{0}^{1}\left(1-t_{1}\right)^{\alpha}\left(1-t_{2}\right)^{\beta} \\
& \quad \times \mid \frac{\partial^{2} f}{\partial t_{1} \partial t_{2}}\left(t_{1} a+\left(1-t_{1}\right) b, t_{2} c\right. \\
& \left.\left.\quad+\left(1-t_{2}\right) d\right) \mid d t_{2} d t_{1}\right\} .
\end{aligned}
$$

Because $\left|\partial^{2} f / \partial u \partial v\right|$ is $s$-convex function on the coordinates on $\Delta$, we obtain

$$
\begin{aligned}
& \mid \frac{f(a, c)+f(a, d)+f(b, c)+f(b, d)}{4} \\
& +\frac{\Gamma(\alpha+1) \Gamma(\beta+1)}{4(b-a)^{\alpha}(d-c)^{\beta}}\left[J_{a^{a^{\prime}, c^{+}}}^{\alpha, \beta} f(b, d)+J_{a^{+}, d^{-}}^{\alpha, \beta} f(b, c)\right. \\
& \left.\quad+J_{b^{-}, c^{+}}^{\alpha, \beta} f(a, d)+J_{b^{-}, d^{-}}^{\alpha, \beta} f(a, c)\right]-A \mid \\
& \leq \frac{(b-a)(d-c)}{4} \\
& \quad \times \iint_{0}^{1}\left[t_{1}^{\alpha} t_{2}^{\beta}+\left(1-t_{1}\right)^{\alpha} t_{2}^{\beta}+t_{1}^{\alpha}\left(1-t_{2}\right)^{\beta}\right. \\
& \left.\quad+\left(1-t_{1}\right)^{\alpha}\left(1-t_{2}\right)^{\beta}\right] \\
& \quad \times\left|\frac{\partial^{2} f}{\partial t_{1} \partial t_{2}}\left(t_{1} a+\left(1-t_{1}\right) b, t_{2} c+\left(1-t_{2}\right) d\right)\right| d t_{2} d t_{1} \\
& \leq \frac{(b-a)(d-c)}{4} \\
& \quad \times \iint_{0}^{1}\left[t_{1}^{\alpha} t_{2}^{\beta}+\left(1-t_{1}\right)^{\alpha} t_{2}^{\beta}+t_{1}^{\alpha}\left(1-t_{2}\right)^{\beta}\right. \\
& \left.+\left(1-t_{1}\right)^{\alpha}\left(1-t_{2}\right)^{\beta}\right]
\end{aligned}
$$




$$
\begin{aligned}
& \times\left\{t_{1}^{s} t_{2}^{s}\left|\frac{\partial^{2} f}{\partial t_{1} \partial t_{2}}(a, c)\right|+\left(1-t_{1}\right)^{s} t_{2}^{s}\left|\frac{\partial^{2} f}{\partial t_{1} \partial t_{2}}(b, c)\right|\right. \\
& +t_{1}^{s}\left(1-t_{2}\right)^{s}\left|\frac{\partial^{2} f}{\partial t_{1} \partial t_{2}}(a, d)\right| \\
& \left.+\left(1-t_{1}\right)^{s}\left(1-t_{2}\right)^{s}\left|\frac{\partial^{2} f}{\partial t_{1} \partial t_{2}}(b, d)\right|\right\} d t_{2} d t_{1} \\
& =\frac{(b-a)(d-c)}{4}\left[\frac{1}{\alpha+s+1}+B(s+1, \alpha+1)\right] \\
& \times\left[\frac{1}{\beta+s+1}+B(s+1, \beta+1)\right]
\end{aligned}
$$

$$
\begin{aligned}
& \times\left[\left|\frac{\partial^{2} f}{\partial t_{1} \partial t_{2}}(a, c)\right|+\left|\frac{\partial^{2} f}{\partial t_{1} \partial t_{2}}(b, c)\right|\right. \\
& \left.+\left|\frac{\partial^{2} f}{\partial t_{1} \partial t_{2}}(a, d)\right|+\left|\frac{\partial^{2} f}{\partial t_{1} \partial t_{2}}(b, d)\right|\right],
\end{aligned}
$$

which completes the proof.

Theorem 13. Let $f: \Delta=[a, b] \times[c, d] \subseteq[0, \infty)^{2} \rightarrow[0, \infty)$ be a partial differentiable mapping with $0 \leq a<b, 0 \leq c<d$. If $\left|\partial^{2} f / \partial u \partial v\right|^{q}$ is s-convex on the coordinates in the second sense on $\Delta$ for some fixed $s \in(0,1]$ and $q>1$. Then, one has the inequalities:

$$
\begin{aligned}
& \mid \frac{f(a, c)+f(a, d)+f(b, c)+f(b, d)}{4}+\frac{\Gamma(\alpha+1) \Gamma(\beta+1)}{4(b-a)^{\alpha}(d-c)^{\beta}} \\
& \quad \times\left[J_{a^{+}, c^{+}}^{\alpha, \beta} f(b, d)+J_{a^{+}, d^{-}}^{\alpha, \beta} f(b, c)+J_{b^{-}, c^{+}}^{\alpha, \beta} f(a, d)\right. \\
& \left.\quad+J_{b^{-}, d^{-}}^{\alpha, \beta}(a, c)\right]-A \mid \\
& \leq \frac{(b-a)(d-c)}{[(\alpha p+1)(\beta p+1)]^{1 / p}} \\
& \quad \times\left(\frac{\left|\left(\partial^{2} f / \partial t_{1} \partial t_{2}\right)(a, c)\right|^{q}+\left|\left(\partial^{2} f / \partial t_{1} \partial t_{2}\right)(b, c)\right|^{q}+\left|\left(\partial^{2} f / \partial t_{1} \partial t_{2}\right)(a, d)\right|^{q}+\left|\left(\partial^{2} f / \partial t_{1} \partial t_{2}\right)(b, d)\right|^{q}}{(s+1)^{2}}\right)^{1 / q},
\end{aligned}
$$

where $(1 / p)+(1 / q)=1$ and $A$ are as given in Lemma 9 .

Proof. From Lemma 9, we obtain

$$
\begin{aligned}
& \mid \frac{f(a, c)+f(a, d)+f(b, c)+f(b, d)}{4}+\frac{\Gamma(\alpha+1) \Gamma(\beta+1)}{4(b-a)^{\alpha}(d-c)^{\beta}} \\
& \times\left[J_{a^{+}, c^{+}}^{\alpha, \beta} f(b, d)+J_{a^{+}, d^{-}}^{\alpha, \beta} f(b, c)+J_{b^{-}, c^{+}}^{\alpha, \beta} f(a, d)\right. \\
& \left.\quad+J_{b^{-}, d^{-}}^{\alpha, \beta} f(a, c)\right]-A \mid \\
& \leq \frac{(b-a)(d-c)}{4} \\
& \quad \times\left\{\iint_{0}^{1} t_{1}^{\alpha} t_{2}^{\beta}\right. \\
& \quad \times\left|\frac{\partial^{2} f}{\partial t_{1} \partial t_{2}}\left(t_{1} a+\left(1-t_{1}\right) b, t_{2} c+\left(1-t_{2}\right) d\right)\right| d t_{2} d t_{1} \\
& +\iint_{0}^{1}\left(1-t_{1}\right)^{\alpha} t_{2}^{\beta} \\
& \quad \times\left|\frac{\partial^{2} f}{\partial t_{1} \partial t_{2}} c\left(t_{1} a+\left(1-t_{1}\right) b, t_{2} c+\left(1-t_{2}\right) d\right)\right| d t_{2} d t_{1}
\end{aligned}
$$

$$
\begin{aligned}
& +\iint_{0}^{1} t_{1}^{\alpha}\left(1-t_{2}\right)^{\beta} \\
& \quad \times\left|\frac{\partial^{2} f}{\partial t_{1} \partial t_{2}}\left(t_{1} a+\left(1-t_{1}\right) b, t_{2} c\left(1-t_{2}\right) d\right)\right| d t_{2} d t_{1} \\
& +\iint_{0}^{1}\left(1-t_{1}\right)^{\alpha}\left(1-t_{2}\right)^{\beta} \\
& \quad \times \mid \frac{\partial^{2} f}{\partial t_{1} \partial t_{2}} \\
& \left.\quad \times\left(t_{1} a+\left(1-t_{1}\right) b, t_{2} c+\left(1-t_{2}\right) d\right) \mid d t_{2} d t_{1}\right\} .
\end{aligned}
$$

By using the well-known Hölder's inequality for double integrals, then one has

$$
\begin{aligned}
& \mid \frac{f(a, c)+f(a, d)+f(b, c)+f(b, d)}{4} \\
& +\frac{\Gamma(\alpha+1) \Gamma(\beta+1)}{4(b-a)^{\alpha}(d-c)^{\beta}}
\end{aligned}
$$




$$
\begin{aligned}
& \times\left[J_{a^{+}, c^{+}}^{\alpha, \beta} f(b, d)+J_{a^{+}, d^{-}}^{\alpha, \beta} f(b, c)\right. \\
& \left.+J_{b^{-}, c^{+}}^{\alpha, \beta} f(a, d)+J_{b^{-}, d^{-}}^{\alpha, \beta} f(a, c)\right]-A \\
& \leq \frac{(b-a)(d-c)}{4}\left\{\left(\iint_{0}^{1} t_{1}^{p \alpha} t_{2}^{p \beta} d t_{2} d t_{1}\right)^{1 / p}\right. \\
& +\left(\iint_{0}^{1}\left(1-t_{1}\right)^{p \alpha} t_{2}^{p \beta} d t_{2} d t_{1}\right)^{1 / p} \\
& +\left(\iint_{0}^{1} t_{1}^{p \alpha}\left(1-t_{2}\right)^{p \beta} d t_{2} d t_{1}\right)^{1 / p} \\
& \left.+\left(\iint_{0}^{1}(1-t)^{p \alpha}(1-s)^{p \beta} d t_{2} d t_{1}\right)^{1 / p}\right\} \\
& \times\left(\iint_{0}^{1} \mid \frac{\partial^{2} f}{\partial t_{1} \partial t_{2}}\left(t_{1} a+\left(1-t_{1}\right) b, t_{2} c\right.\right. \\
& \left.\left.+\left(1-t_{2}\right) d\right)\left.\right|^{q} d t_{2} d t_{1}\right)^{1 / q} . \\
& \iint_{0}^{1}\left|\frac{\partial^{2} f}{\partial t_{1} \partial t_{2}}\left(t_{1} a+\left(1-t_{1}\right) b, t_{2} c+\left(1-t_{2}\right) d\right)\right|^{q} d t_{2} d t_{1} \\
& \leq \frac{\left|\left(\partial^{2} f / \partial t_{1} \partial t_{2}\right)(a, c)\right|^{q}+\left|\left(\partial^{2} f / \partial t_{1} \partial t_{2}\right)(b, c)\right|^{q}+\left|\left(\partial^{2} f / \partial t_{1} \partial t_{2}\right)(a, d)\right|^{q}+\left|\left(\partial^{2} f / \partial t_{1} \partial t_{2}\right)(b, d)\right|^{q}}{(s+1)^{2}} .
\end{aligned}
$$

So

$$
\begin{aligned}
& \mid \frac{f(a, c)+f(a, d)+f(b, c)+f(b, d)}{4}+\frac{\Gamma(\alpha+1) \Gamma(\beta+1)}{4(b-a)^{\alpha}(d-c)^{\beta}} \\
& \quad \times\left[J_{a^{+}, c^{+}}^{\alpha, \beta} f(b, d)+J_{a^{+}, d^{-}}^{\alpha, \beta} f(b, c)+J_{b^{-}, c^{+}}^{\alpha, \beta} f(a, d)+J_{b^{-}, d^{-}}^{\alpha, \beta} f(a, c)\right]-A \mid \\
& \leq \frac{(b-a)(d-c)}{[(\alpha p+1)(\beta p+1)]^{1 / p}} \\
& \quad \times\left(\frac{\left|\left(\partial^{2} f / \partial t_{1} \partial t_{2}\right)(a, c)\right|^{q}+\left|\left(\partial^{2} f / \partial t_{1} \partial t_{2}\right)(b, c)\right|^{q}+\left|\left(\partial^{2} f / \partial t_{1} \partial t_{2}\right)(a, d)\right|^{q}+\left|\left(\partial^{2} f / \partial t_{1} \partial t_{2}\right)(b, d)\right|^{q}}{(s+1)^{2}}\right)^{1 / q},
\end{aligned}
$$

which completes the proof.

Theorem 14. Let $f: \Delta=[a, b] \times[c, d] \subseteq[0, \infty)^{2} \rightarrow[0, \infty)$ be a partial differentiable mapping with $0 \leq a<b, 0 \leq c<d$. If $\left|\partial^{2} f / \partial u \partial \nu\right|^{q}$ is s-concave on the coordinates in the second sense on $\Delta$ for some fixed $s \in(0,1]$ and $q>1$, then one has the inequalities:

$$
\begin{aligned}
& \mid \frac{f(a, c)+f(a, d)+f(b, c)+f(b, d)}{4}+\frac{\Gamma(\alpha+1) \Gamma(\beta+1)}{4(b-a)^{\alpha}(d-c)^{\beta}} \\
& \times\left[J_{a^{+}, c^{+}}^{\alpha, \beta} f(b, d)+J_{a^{+}, d^{-}}^{\alpha, \beta} f(b, c)\right. \\
& \left.\quad+J_{b^{-}, c^{+}}^{\alpha, \beta} f(a, d)+J_{b^{-}, d^{-}}^{\alpha, \beta} f(a, c)\right]-A \mid \\
& \leq \frac{(b-a)(d-c)}{[(\alpha p+1)(\beta p+1)]^{1 / p}} \\
& \quad \times 4^{(s-1) / q}\left|\frac{\partial^{2} f}{\partial t_{1} \partial t_{2}}\left(\frac{a+b}{2}, \frac{c+d}{2}\right)\right|,
\end{aligned}
$$

where $(1 / p)+(1 / q)=1$ and $A$ is as given in Lemma 9 .

Proof. Similarly as in Theorem 13, we obtain

$$
\begin{aligned}
& \mid \frac{f(a, c)+f(a, d)+f(b, c)+f(b, d)}{4} \\
& +\frac{\Gamma(\alpha+1) \Gamma(\beta+1)}{4(b-a)^{\alpha}(d-c)^{\beta}} \\
& \times\left[J_{a^{+}, c^{+}}^{\alpha, \beta} f(b, d)+J_{a^{+}, d^{-}}^{\alpha, \beta} f(b, c)\right. \\
& \left.\quad+J_{b^{-}, c^{+}}^{\alpha, \beta} f(a, d)+J_{b^{-}, d^{-}}^{\alpha, \beta} f(a, c)\right]-A \mid \\
& \leq \frac{(b-a)(d-c)}{4} \\
& \times\left\{\left(\iint_{0}^{1} t_{1}^{p \alpha} t_{2}^{p \beta} d t_{2} d t_{1}\right)^{1 / p}\right. \\
& +\left(\iint_{0}^{1}\left(1-t_{1}\right)^{p \alpha} t_{2}^{p \beta} d t_{2} d t_{1}\right)^{1 / p}
\end{aligned}
$$




$$
\begin{gathered}
+\left(\iint_{0}^{1} t_{1}^{p \alpha}\left(1-t_{2}\right)^{p \beta} d t_{2} d t_{1}\right)^{1 / p} \\
\left.+\left(\iint_{0}^{1}(1-t)^{p \alpha}(1-s)^{p \beta} d t_{2} d t_{1}\right)^{1 / p}\right\} \\
\times\left(\iint_{0}^{1} \mid \frac{\partial^{2} f}{\partial t_{1} \partial t_{2}}\left(t_{1} a+\left(1-t_{1}\right) b, t_{2} c\right.\right. \\
\left.\left.+\left(1-t_{2}\right) d\right)\left.\right|^{q} d t_{2} d t_{1}\right)^{1 / q}
\end{gathered}
$$

Because $\left|\partial^{2} f / \partial t_{1} \partial t_{2}\right|$ is $s$-concave function on the coordinates on $\Delta$, by the reversed direction of (8), then one has

$$
\begin{aligned}
& \iint_{0}^{1}\left|\frac{\partial^{2} f}{\partial t_{1} \partial t_{2}}\left(t_{1} a+\left(1-t_{1}\right) b, t_{2} c+\left(1-t_{2}\right) d\right)\right|^{q} d t_{2} d t_{1} \\
& \quad \leq 4^{s-1}\left|\frac{\partial^{2} f}{\partial t_{1} \partial t_{2}}\left(\frac{a+b}{2}, \frac{c+d}{2}\right)\right|^{q} .
\end{aligned}
$$

Then

$$
\begin{aligned}
& \mid \frac{f(a, c)+f(a, d)+f(b, c)+f(b, d)}{4}+\frac{\Gamma(\alpha+1) \Gamma(\beta+1)}{4(b-a)^{\alpha}(d-c)^{\beta}} \\
& \quad \times\left[J_{a^{+}, c^{+}}^{\alpha, \beta} f(b, d)+J_{a^{+}, d^{-}}^{\alpha, \beta} f(b, c)\right. \\
& \left.\quad+J_{b^{-}, c^{+}}^{\alpha, \beta} f(a, d)+J_{b^{-}, d^{-}}^{\alpha, \beta} f(a, c)\right]-A \mid \\
& \leq \frac{(b-a)(d-c)}{[(\alpha p+1)(\beta p+1)]^{1 / p}} \\
& \quad \times 4^{(s-1) / q}\left|\frac{\partial^{2} f}{\partial t_{1} \partial t_{2}}\left(\frac{a+b}{2}, \frac{c+d}{2}\right)\right| .
\end{aligned}
$$

We get the desired results.

\section{Conclusion}

In this paper, we obtain some Hermite-Hadamard type inequalities for coordinated $s$-convex functions via RiemannLiouville integrals. An interesting topic is whether we can use the methods in this paper to establish the HermiteHadamard inequalities for other kinds of convex functions on the coordinates via Riemann-Liouville integrals.

\section{Conflict of Interests}

The author declares that there is no conflict of interests regarding the publication of this paper.

\section{Acknowledgment}

This work is supported by Youth Project of Chongqing Three Gorges University of China (no. 13QN11).

\section{References}

[1] G. Farid, S. Abramovich, and J. Pečarić, "More about HermiteHadamard inequalities, Cauchy's means, and superquadracity," Journal of Inequalities and Applications, vol. 2010, Article ID 102467, 14 pages, 2010.

[2] M. Bessenyei and Z. Páles, "Hadamard-type inequalities for generalized convex functions," Mathematical Inequalities \& Applications, vol. 6, no. 3, pp. 379-392, 2003.

[3] S. S. Dragomir and S. Fitzpatrick, "The Hadamard inequalities for $s$-convex functions in the second sense," Demonstratio Mathematica, vol. 32, no. 4, pp. 687-696, 1999.

[4] S. S. Dragomir, "On the Hadamard's inequlality for convex functions on the co-ordinates in a rectangle from the plane," Taiwanese Journal of Mathematics, vol. 5, no. 4, pp. 775-788, 2001.

[5] A. El Farissi, "Simple proof and refinement of HermiteHadamard inequality," Journal of Mathematical Inequalities, vol. 4, no. 3, pp. 365-369, 2010.

[6] X. Gao, "A note on the Hermite-Hadamard inequality," Journal of Mathematical Inequalities, vol. 4, no. 4, pp. 587-591, 2010.

[7] M. Z. Sarikaya, E. Set, and H. Yildirim, "On some Hadamardtype inequalities for $h$-convex functions," Journal of Mathematical Inequalities, vol. 2, no. 3, pp. 335-341, 2008.

[8] H. Hudzik and L. Maligranda, "Some remarks on s-convex functions," Aequationes Mathematicae, vol. 48, no. 1, pp. 100111, 1994.

[9] M. Alomari and M. Darus, "The Hadamard's inequality for $s$ convex function of 2-variables on the co-ordinates," International Journal of Mathematical Analysis, vol. 2, no. 13, pp. 629638, 2008.

[10] M. Alomari and M. Darus, "Hadamard-type inequalities for $s$ convex functions," International Mathematical Forum, vol. 3, no. 40, pp. 1965-1975, 2008.

[11] M. Z. Sarikaya, E. Set, H. Yaldiz, and N. Başak, "HermiteHadamard's inequalities for fractional integrals and related fractional inequalities," Mathematical and Computer Modelling, vol. 57, no. 9-10, pp. 2403-2407, 2013.

[12] M. Z. Sarıkaya, "On the Hermite-Hadamard-type inequalities for co-ordinated convex function via fractional integrals," Integral Transforms and Special Functions, vol. 25, no. 2, pp. 134-147, 2014. 


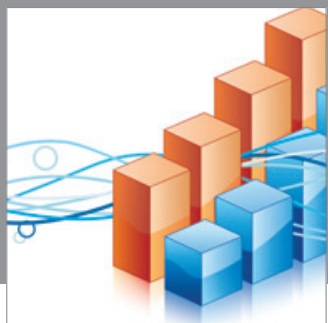

Advances in

Operations Research

mansans

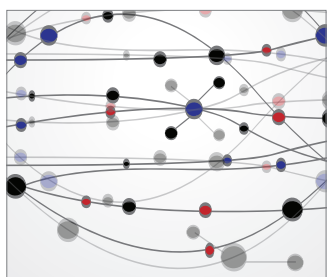

The Scientific World Journal
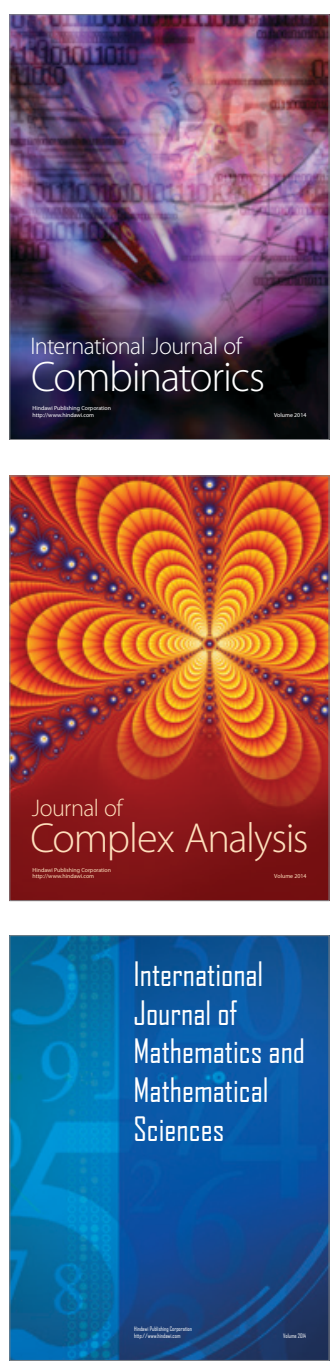
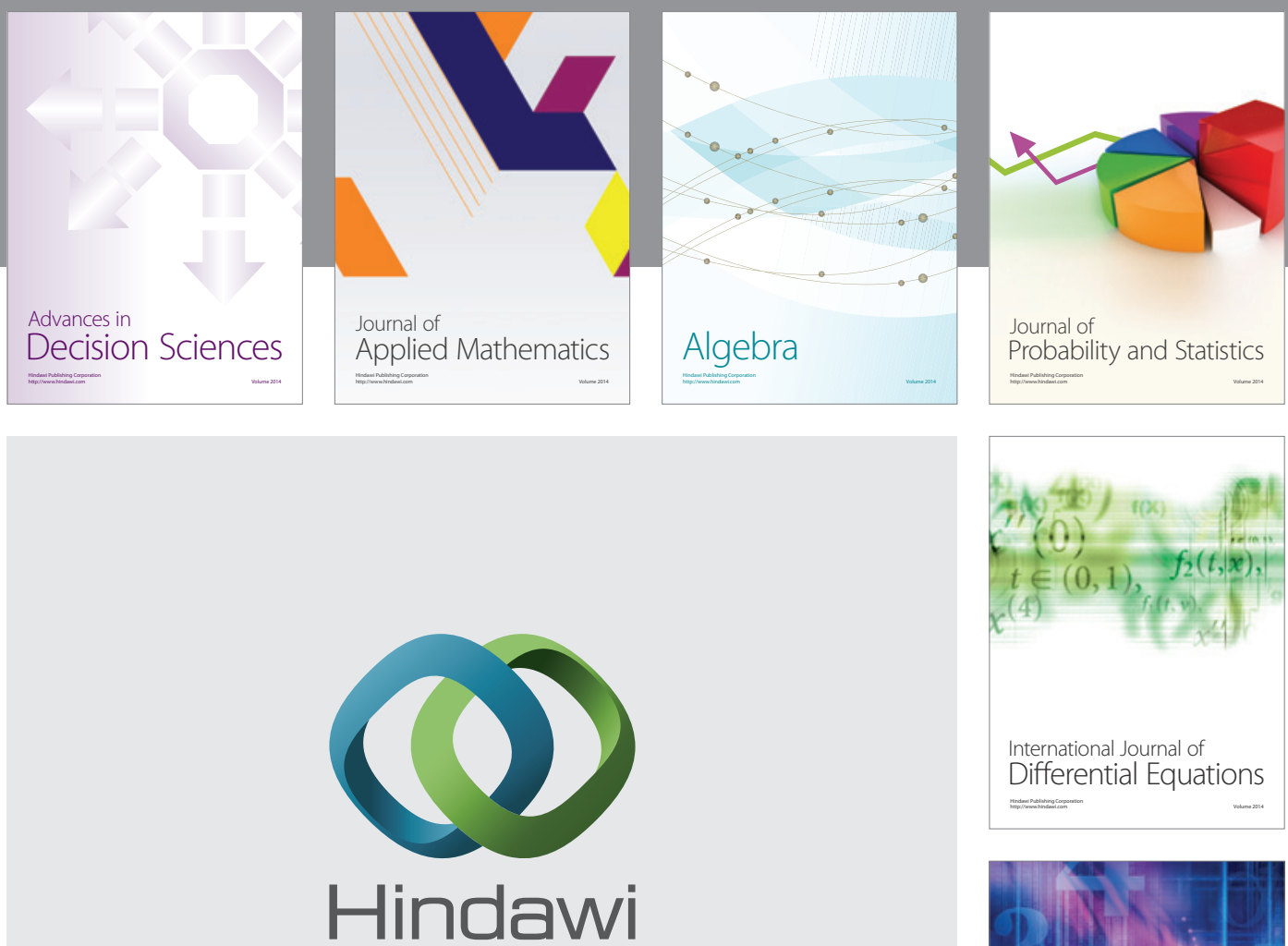

Submit your manuscripts at http://www.hindawi.com
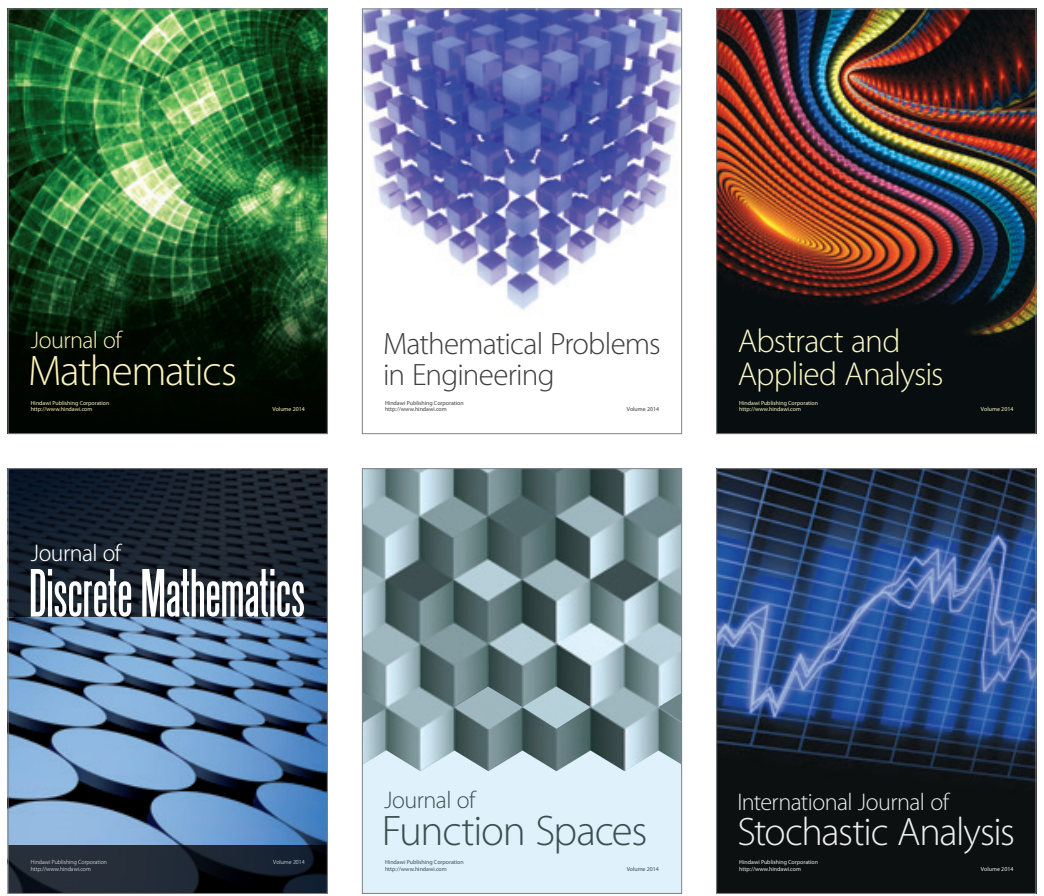

Journal of

Function Spaces

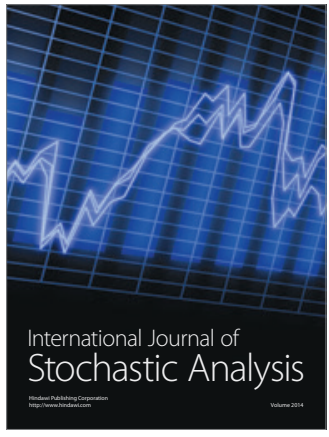

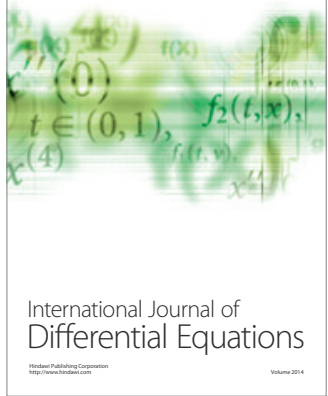
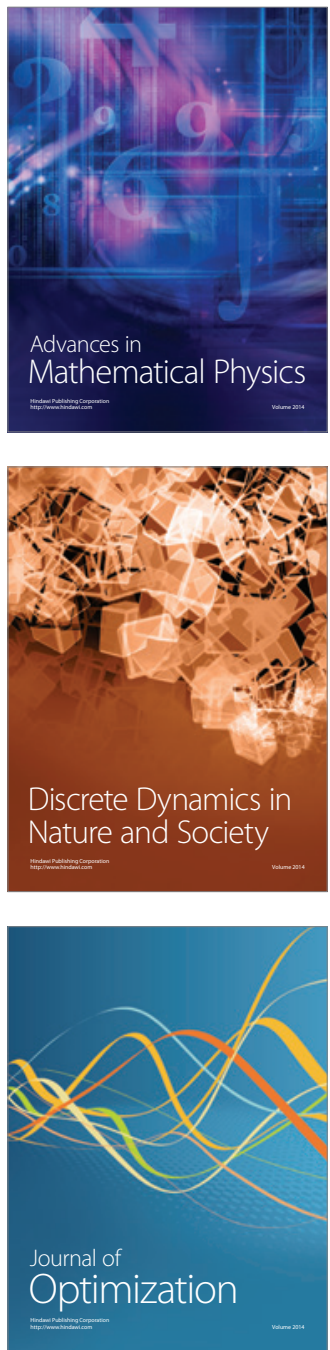